\title{
Improving Conical Scanning for Satellite Tracking On-The-Move
}

\author{
Robert Karol ${ }^{1}$, Gunnar Ristroph ${ }^{2}$ \\ ${ }^{1}$ California Institute of Technology, Pasadena, California USA \\ ${ }^{2}$ IJK Controls LLC, Dallas, Texas USA
}

\begin{abstract}
Mobile satellite antennas are gimbal-mounted with an active control system to keep the antenna pointed at the target satellite. Short time-scale antenna stabilization is achieved with gyroscope feedback. Long time-scale tracking of the satellite is maintained by scanning the antenna: The beam is intentionally nutated and the resulting change in signal strength is used to estimate how well the antenna is aligned to the satellite. We review the basic architectures for tracking with an outer control loop, assuming that the system is built upon a fast inner stabilization loop. The problem is formulated in a generic mathematical way, not specific to RF satellite communication. Approaches and nomenclature from other fields faced with the same problem of peak-seeking control are discussed. One common and simple technique for tracking is analyzed in detail to reveal the shortcomings that lead to degraded performance. An improvement is suggested and subjected to the same transient analysis to show the improvement. Implications to the overall closed-loop tracking systems are discussed.
\end{abstract}

Keywords: satellite tracking, satcom on-the-move, peak-seeking control, conical scanning, nutating beam, stabilized gimbal tracking

\section{INTRODUCTION}

Mobile satellite communications systems must keep an antenna pointed as close as possible at a far target, the satellite. The satellite is not moving in inertial space. By inertially stabilizing the antenna with gyroscope feedback, pointing can be maintained over short time-scales. Over long time scales, however, gyroscope errors and unrejected base disturbances cause the antenna to not point correctly. When mis-pointed, the received signal strength is reduced.

In order to maintain proper pointing over long time-scales, many control systems intentionally dither the antenna and observe the resulting change in signal strength to determine which way to move the antenna. The intuition is simple: If the antenna is intentionally moved to the right and the signal strength improved, this must mean that the satellite is to the right. Conversely, if the signal strength is weakened by moving to the right, the satellite must be to the left. This process of dithering, nutating, scanning, or perturbing the antenna and using the resulting change in signal strength to determine where to point is referred to as "tracking" or keeping the signal "peaked up." 1

The basic problem - keeping a signal "peaked up" by using a measurement of the signal itself, without a direct measurement of the two-dimensional position relative to the ideal - is similar to problems in other fields, unrelated to communication, pointing or gimbal systems.

\section{SIMPLIFIED PROBLEM STATEMENT}

An actual implementation of a stabilized, tracking satellite antenna has many practical concerns that must be addressed: gyroscope noise, antenna beam shape, atmospheric impacts on signal propagation, etc. In order to simplify the problem to its fundamental essence, we formulate the problem in a more mathematically pure way.

Figure 1 shows this simplified formulation in a Cartesian plane. We have measurements (albeit with noise) of $\dot{x}$ and $\dot{y}$. These correspond to inertial rates as reported by the gyroscopes. We have a measurement (albeit with noise) $R^{2}=x^{2}+y^{2}$ of this range to the target. These correspond to a measure provided by the received signal strength. Our actuators and external disturbances produce accelerations in $x$ and $y$.

We have no direct measurement of $x$ and $y$ - just of $\dot{x}, \dot{y}$, and $x^{2}+y^{2}$.

Further author information: E-mail: info@ijkco.com 


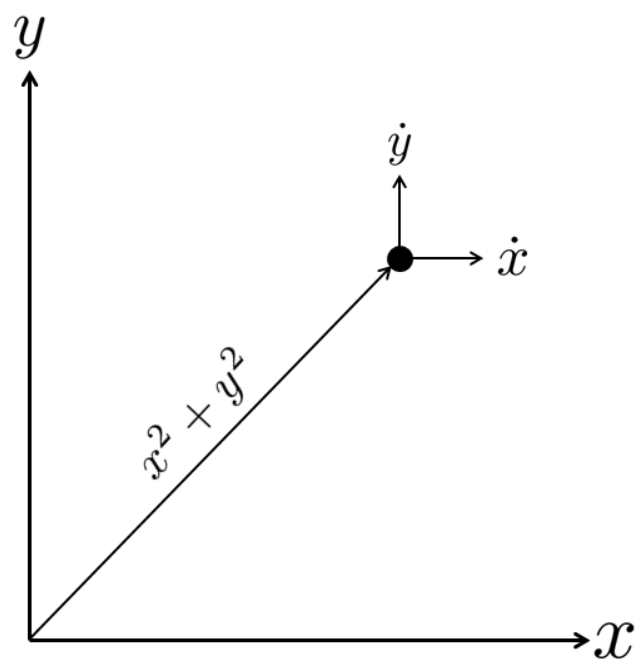

Figure 1. The problem is formulated in a simplified Cartesian plane. Although subtleties and practical considerations are ignored, this formulation captures the essential nature of the problem: velocity measurements, a single range measurement and no position measurement.

All of our measurements are, of course, noisy. In practice, our measurement of $x^{2}+y^{2}$ is particularly noisy and this is one of the reasons that the tracking can only occur over long time-scales (low bandwidth).

\section{OVERVIEW OF APPROACH ALTERNATIVES}

Although it isn't necessary, all solutions that we consider have inner stabilization loops for $\dot{x}$ and $\dot{y}$. All solutions that we consider use a circular scan in $x$ and $y$. This appears as a cone using a 3D gimbal and so is referred to as "conical scan" or "conscan."

Most of these approaches follow the basic block diagram shown in figure 2. Note that these approaches implicitly or explicitly produce some estimate of position $x$ and $y$. In what we call the Classic Demodulation Conscan approach, the perturbation signal (as viewed in position space) is correlated with the range measurement to estimate position as shown in figure 3. The formulation and analysis was developed for NASA/JPL Deep Space Network antennas. ${ }^{2}$ Although the application was for fixed Earth stations (not mobile, with no stabilization), the tracking algorithm is the same as is used on many mobile antennas. NASA/JPL have since researched and implemented a variety of other tracking techniques. Rather than using a naïve correlation, these more advanced architectures use a Kalman filter formulation for the estimator.

The general problem of finding and staying at a peak is called "peak-seeking" throughout the general control literature, although this term is not used much in the gimbal pointing or tracking industry. An example of a similar problem comes from formation flight control. Two aircraft seek to maximize the aerodynamic efficiency (reduce drag) by flying close to one another. The aerodynamic efficiency is maximized at a particular relative position, which cannot be known exactly a priori and may move slowly with changing parameters.

The controls architecture is similar to conscanning: a scanning perturbation is added into the signal for the low-level stabilization loop*. A measure of the formation efficiency is monitored over long time scales by a Kalman filter based estimator that produces an estimate of the position. ${ }^{3}$

\footnotetext{
*Unfortunately, the formation flight literature calls the fast, inner velocity loop a "tracking" loop and provides no name for the outer position compensation. We call the fast, inner velocity loop a "stabilization" loop.
} 


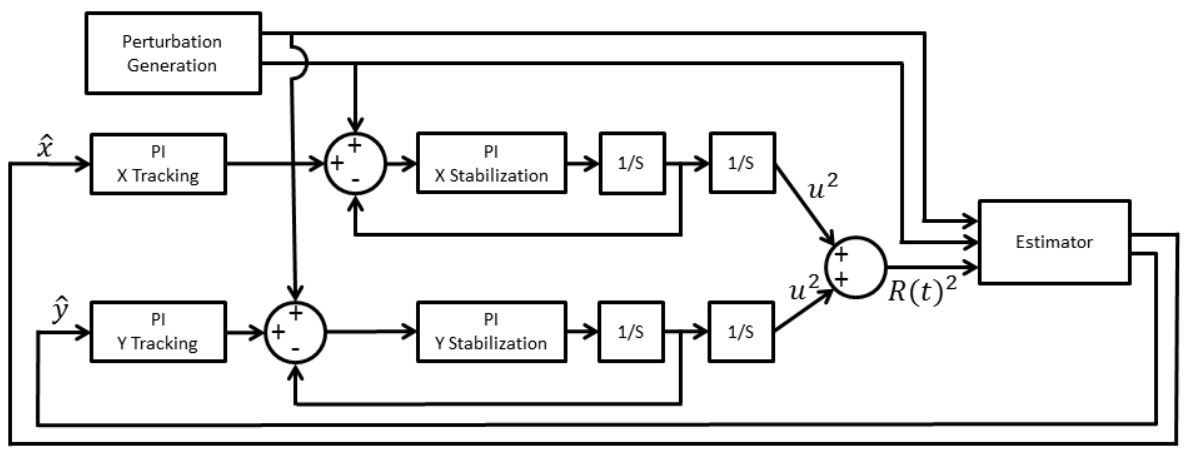

Figure 2. Both axes have an inner, fast stabilization loop. The outer tracking loop compensation acts to keep the system at $x=0, y=0$ by commanding the stabilization (velocity) loop. The perturbation generation commands the inner loop as well and creates the dithering or scanning motion. The Estimator observes the scanning action and $x^{2}+y^{2}$ to estimate position for the outer loop to use.

Equations 1 and 2 show the position perturbation around the boresight axis induced by moving the dish with sinusoidal signals in both the $\mathrm{x}$-and $\mathrm{y}$ axis. The circular scanning motion is characterized by two parameters: the frequency $(\omega)$ and amplitude $(A)$. The frequency clearly should not be beyond the stabilization loop bandwidth because then the stabilization loop would not be able to keep up with the commanded perturbation motion. Increasing the amplitude increases the effective signal to noise ratio, but is undesirable because it means that the antenna spends more time away from the ideal position.

$$
\begin{aligned}
& x_{\text {perturb }}(t)=A \cos (\omega t+\phi) \\
& y_{\text {perturb }}(t)=A \sin (\omega t+\phi)
\end{aligned}
$$

In the steady-state with no noise, by adding these perturbations to our initial position $\left(x_{0}, y_{0}\right)$ we get equations 3 and 4 showing the location the dish is pointing over all time.

$$
\begin{aligned}
x(t) & =x_{0}+x_{\text {perturb }}(t)=x_{0}+A \cos (\omega t+\phi) \\
y(t) & =y_{0}+y_{\text {perturb }}(t)=y_{0}+A \sin (\omega t+\phi)
\end{aligned}
$$

Combining these two equations gives:

$$
R(t)^{2}=x(t)^{2}+y(t)^{2}=\left(x_{0}+A \cos (\omega t+\phi)\right)^{2}+\left(y_{0}+A \sin (\omega t+\phi)\right)^{2}
$$

\section{CLASSIC DEMODULATION CONSCAN}

\subsection{Estimator}

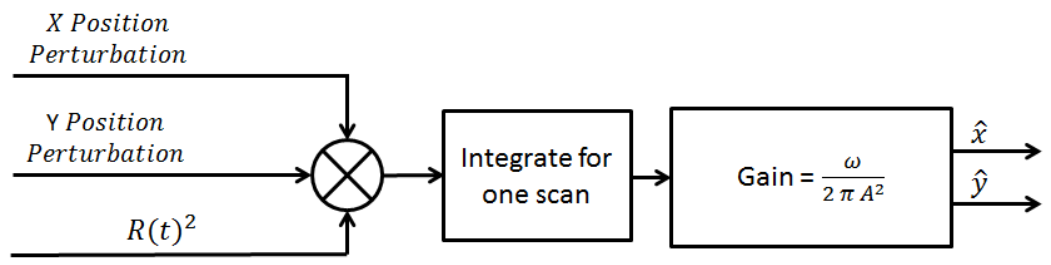

Figure 3. The range measurement (signal strength) is convolved with the position perturbation. 
Under the traditional method of conical scanning, the signal strength $R^{2}$ is convolved with the position perturbation $\left(x_{\text {perturb }}, y_{\text {perturb }}\right)$ and integrated over a full scan in order to get a position estimate $(\hat{x}, \hat{y})$. Figure 3 shows an overview of the estimator which takes in the received signal and convolves it with the position perturbation signals in order to get an estimate of the current position.

Equations 6 and 7 show the most general form of the estimator.

$$
\begin{aligned}
& \hat{x}(t)=\frac{\omega}{2 \pi A^{2}} \int_{t-\frac{2 \pi}{\omega}}^{t} R(\tau)^{2} x_{\text {perturb }}(\tau) \mathrm{d} \tau \\
& \hat{y}(t)=\frac{\omega}{2 \pi A^{2}} \int_{t-\frac{2 \pi}{\omega}}^{t} R(\tau)^{2} y_{\text {perturb }}(\tau) \mathrm{d} \tau
\end{aligned}
$$

Maintaining the assumption that the system is at steady state without noise, it becomes possible to substitute in equations 5,1 , and 2 to get equations 8 , and 9 . These equations show that with the gain factor of $\frac{\omega}{2 \pi A^{2}}$ was chosen so that $\hat{x}=x_{0}$ and $\hat{y}=y_{0}$ after a single cycle.

$$
\begin{aligned}
& \hat{x}(t)=\frac{\omega}{2 \pi A^{2}} \int_{t-\frac{2 \pi}{\omega}}^{t}\left(\left(A \cos (\omega \tau+\phi)+x_{0}\right)^{2}+\left(A \sin (\omega \tau+\phi)+y_{0}\right)^{2}\right) A \cos (\omega \tau+\phi) d \tau=x_{0} \\
& \hat{y}(t)=\frac{\omega}{2 \pi A^{2}} \int_{t-\frac{2 \pi}{\omega}}^{t}\left(\left(A \cos (\omega \tau+\phi)+x_{0}\right)^{2}+\left(A \sin (\omega \tau+\phi)+y_{0}\right)^{2}\right) A \sin (\omega \tau+\phi) d \tau=y_{0}
\end{aligned}
$$

\subsection{Step Response}

While the steady state response of the estimator is perfect, an actual system will be moving through a combination of controlled motion during tracking, and noise introduced into the system by external torques. In order to determine the effects of this motion it becomes important to analyze the transient response. Figure 4 shows the step response of the estimator when scanning with a few different radii.

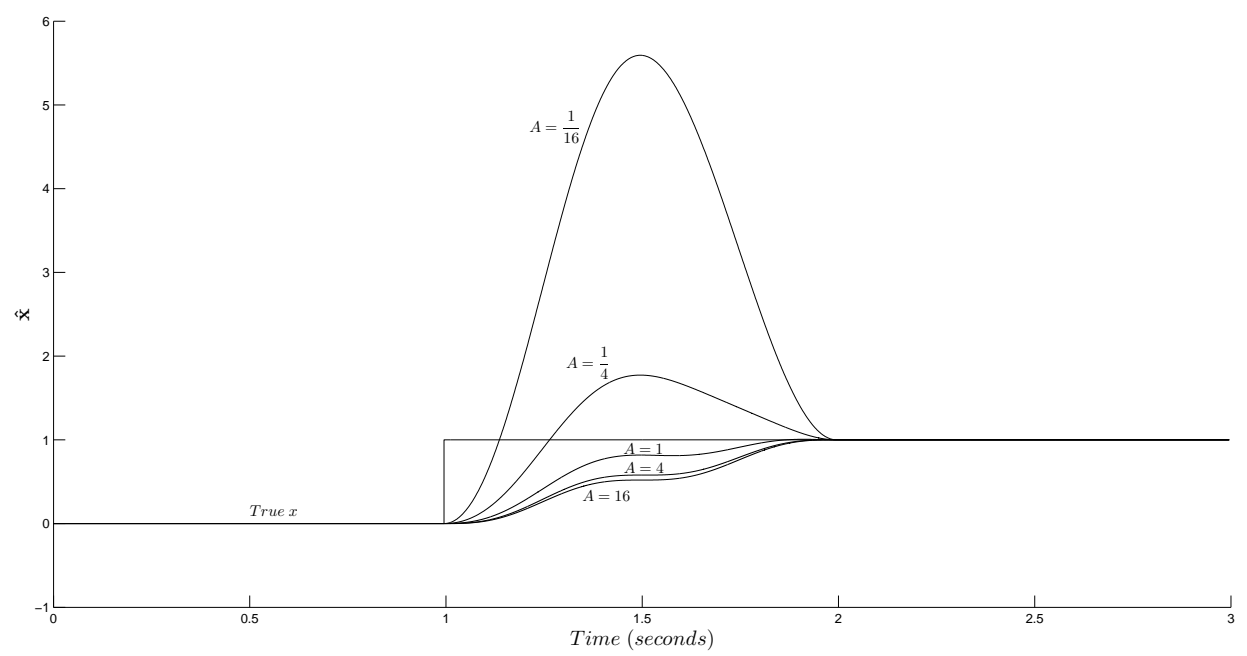

Figure 4. Step response of the old estimator with $\omega=2 \pi$ and $\phi=\frac{3 \pi}{2}$ with varying $A$. The shape of response depends on where within the scan cycle the step occurred and this is captured by $\phi$. These plots use one value of $\phi$ for illustration of the nature of the step response. 


\subsection{Problems}

The traditional conical scanning estimator works very well under conditions where the radius of the scan is comparable to or larger than the motion caused by tracking or external noises, however, figure 4 shows how far off the estimator can be when the radius of the scan is too small. While this problem could be overcome by using a large scanning radius, larger scans prevent the dish from pointing directly at the origin since during the scan, the dish is constantly being forced away from the best estimate by a distance equal to the radius of the scan.

\section{DERIVATIVE DEMODULATION CONSCAN}

\subsection{Estimator}

A new estimator was designed to help eliminate some of the problems that arose using the traditional method many of which are due to the fact that the $R^{2}$ term is unbounded and increases quickly while moving away from the origin.

Rather than using the value of the received signal strength $R^{2}(t)$ directly, the new conical scanning estimator uses the derivative of the received signal $\frac{d}{d t}\left[R(t)^{2}\right]$ strength, and convolves it with the velocity perturbation signal $\left(\frac{d}{d t}\left[x_{\text {perturb }}\right], \frac{d}{d t}\left[y_{\text {perturb }}\right]\right)$ over one scan in order to estimate the current position. Figure 5 shows a schematic for the new estimator which has a different gain, as well as different signals to convolve.

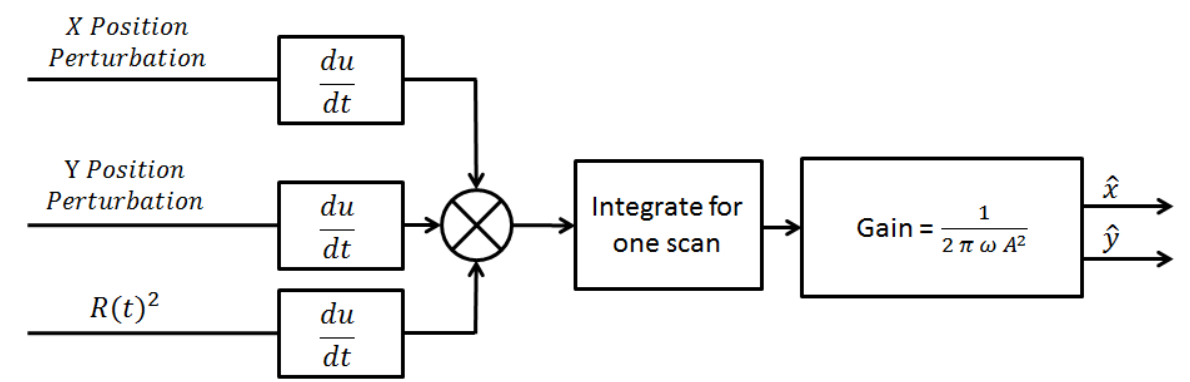

Figure 5. The range measurement (signal strength) is differentiated with respect to time before being convolved with the velocity perturbation. Note that this convolution is effectively happening in rate space as opposed to position space.

The most general form of the new estimator is given by equations 10 and 11 .

$$
\begin{aligned}
& \hat{x}(t)=\frac{1}{2 \pi A^{2} \omega} \int_{t-\frac{2 \pi}{\omega}}^{t} \frac{d}{d \tau}\left[R(\tau)^{2}\right] \frac{d}{d \tau}\left[x_{\text {perturb }}(\tau)\right] \mathrm{d} \tau \\
& \hat{y}(t)=\frac{1}{2 \pi A^{2} \omega} \int_{t-\frac{2 \pi}{\omega}}^{t} \frac{d}{d \tau}\left[R(\tau)^{2}\right] \frac{d}{d \tau}\left[y_{\text {perturb }}(\tau)\right] \mathrm{d} \tau
\end{aligned}
$$

As with the traditional conical scanning estimator, by making the assumption that the system is at steady state with no noise, we can substitute equations 5, 1, and 2 into the estimator. After substituting in equations 5,1 , and 2 we get equations 12 and 13 and find that this estimator is also able to perfectly estimate position after a single cycle.

$$
\begin{aligned}
& \hat{x}(t)=\frac{1}{2 \pi A^{2} \omega} \int_{t-\frac{2 \pi}{\omega}}^{t} \frac{d}{d \tau}\left[\left(A \cos (\omega \tau)+x_{0}\right)^{2}+\left(A \sin (\omega \tau)+y_{0}\right)^{2}\right](-A \omega \sin (\omega \tau)) \mathrm{d} \tau=x_{0} \\
& \hat{y}(t)=\frac{1}{2 \pi A^{2} \omega} \int_{t-\frac{2 \pi}{\omega}}^{t} \frac{d}{d \tau}\left[\left(A \cos (\omega \tau)+x_{0}\right)^{2}+\left(A \sin (\omega \tau)+y_{0}\right)^{2}\right](A \omega \cos (\omega \tau)) \mathrm{d} \tau=y_{0}
\end{aligned}
$$




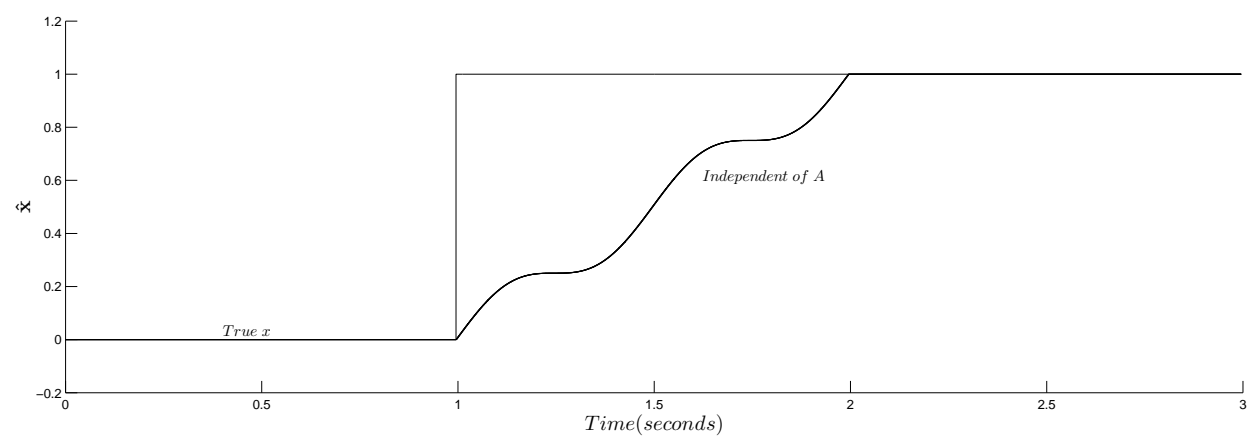

Figure 6. Step response of the new estimator with $\omega=2 \pi$ and $\phi=\frac{3 \pi}{2}$. The shape of response depends on the where within the scan cycle the step occurred. This is captured by $\phi$. In this case, the step response does not depend on the value of $A$, which captures the relationship between the scan size and the step.

\subsection{Step Response}

The step response for the new estimator can be seen in figure 6 with the same properties used in figure 4 .

Using this new estimator, the step response is independent of the conical scanning radius, and monotonically increasing over a single cycle. The estimator no longer estimates a position which is significantly different from the true position. Additionally, since the step response is independent of $A$ a smaller conical scanning radius can be used which keeps the dish pointed closer to the estimated position and improves the overall received signal strength.

\section{CONCLUSION}

Tracking performance can be improved by increasing the bandwidth of the tracking compensation shown in figure 2. Since the conscanning correlation introduces a fundamental phase loss, the track loop bandwidth cannot be close to the conscan frequency $(\omega)$ without losing system stability. Further, the transient behavior from the classical conscan demod approach, shown in figure 4, introduces a growing signal that can cause the system to go unstable. The transient behavior shown by the improved approach, shown in figure 6 , is much less likely to cause the system to go unstable, meaning the tracking loop bandwidth can be increased further. This has been verified in full closed-loop simulations of the simplified system discussed.

The particular architecture improvement suggested here may result in increased performance by enabling increased track loop bandwidth. Other metrics considered during the analysis were noise sensitivity and crossaxis sensitivity. Although the noise performance of the suggested new estimator was worse, this does not significantly impact overall system pointing performance because the track loop bandwidth in either case is so low.

Many other architectures can be considered. Perturbation need not be circular and could be random. Because the line-of-sight already has gyroscopes on it, the gyroscope signals themselves could be used in the estimation as opposed to the perturbation signals. The perturbation signal could be dynamically adjusted to increase observability only when needed.

Improved tracker performance from architectural and algorithmic changes lead directly to improved performanceor, alternatively, decreased cost by allowing the use of lower accuracy inertial sensing. This area is ripe for improvement and could benefit from increased formalization and documentation.

\section{REFERENCES}

[1] DeBruin, J., "Control systems for mobile satcom antennas," IEEE Control Systems Magazine 28, 86-101 (2008). 
[2] Ohlson, J. and Reid, M., "Conical-scan tracking with the 64-m-diameter antenna at goldstone," NASA Technical Report 32-1605, Jet Propulsion Laboratory, California Institute of Technology 32 (1976).

[3] Chichka, Speyer, F. and Park, "Peak-seeking control for drag reduction in formation flight," AIAA J. Guidance, Control, and Dynamics 29, 1221-1230 (2006). 